\title{
31st Meeting of the \\ Canadian Congress of Neurological Sciences Scientific Program
}

\section{GUESTS OF THE CONGRESS}

\author{
Speaker of the Royal College of Physicians and Surgeons - Neurology: \\ What do Experimental Models of Migraine Teach Us About Its Treatment \\ Speaker of the Royal College of Physicians and Surgeons - Neurosurgery \\ A Tour Around the Craniocervical Junction \\ Speaker of the Royal College of Physicians and Surgeons - Paediatric \\ Neurology: Peroxisomal Disorders: Genetics and Therapy \\ Richardson Lecture: Functional Activation and PET \\ Penfield Lecture: The Surgical Treatment of Limbic and Paralimbic \\ Tumours \\ Canadian Society of Clincical Neurophysiologists Guest Lecture: The \\ Decision to Make a Movement - Neurophysiological Insights
}

\author{
Peter Goadsby (London, England) \\ Arnold Menezes (Iowa City) \\ Hugo Moser (Baltimore) \\ R. Frackowiak (London, England) \\ M. Gazi Yazargil (Little Rock)
}

Michael Aminoff (San Francisco)

\section{Child Neurology Day}

\section{Focal Epilepsies}

This course is intended to improve understanding of the relationship underlying focal pathology to the clinical manifestations (i.e. clinical symptomatology predicts site of origin of seizure, congruence of clinical manifestation to electroencephalographic data to neuroimaging data), to develop an understanding of the underlying pathophysiology of focal seizures (i.e. why focal seizures are precipitated and why they do and do not become secondarily generalised), and to enable the classification of focal seizures according to the ILAE (1989) classification with emphasis on appropriate investigation and the cost effectiveness of various investigations. As well attendees will learn something of more recent investigative techniques and their role in further elucidating the pathophysiology of focal seizure and their role in clinical management.

\section{Clinico-Pathological Conference}

Participants will learn an approach to the diagnosis of neurodegenerative disease from a recognized expert in the area and will participate in a group discussion.

\section{Anniversary}

The session will take the form of three interview 'panels' each with an interviewer who will lead the discussion. The panels will reflect the early days of the $\mathrm{CACN}$, its current standing and its future. The session is intended to bring the history of the $\mathrm{CACN}$ to life and inform members about the achievements of the CACN and its members, to enable members of the CACN to reflect on the purpose of the $\mathrm{CACN}$ and its future role in the medical community and society, and to allow open discussion about the current state of Pediatric Neurology in Canada and the role of the CACN.

\section{Pre-Congress Symposia}

\section{Advances in Stroke}

The general objective of this course will be to update participating physicians in the investigation and treatment of cerebrovascular disease. The specific aim is to provide very recent diagnostic and therapeutic information to practicing neurologists about recent therapies in the treatment of acute cerebral ischemia using several new compounds. The clinical indications and contra-indications of newer pharmacological approaches will be reviewed as well as newer investigational techniques. Finally, an update will be given on the risks and benefits of carotid surgery in stroke prevention. The above will hopefully help neurologists to manage more efficiently, in their own practice, patients suffering from cerebral ischemia as well as other types of hemorrhagic cerebrovascular disorders. The topics have a very practical impact and are being covered by experts in their own field. Neurologists attending the symposium should be able to utilize this new knowledge in their daily practices as stroke is one of the most common conditions treated by neurologists.

\section{Advances in the Diagnostic and Treatment Assessment of Alzheimer Disease}

The advent of symptomatic therapy for Alzheimer's disease has underscored the need for improved biological and clinical markers. Recent developments in the sphere of biological markers indicating that the apolipoprotein genotype might have a certain utility in identifying risks for the disease requires that practicing clinicians are well familiar with these types of tests. Furthermore, most recent developments have claimed that specific apolipoprotein subgroups may be more responsive to symptomatic therapy than others. Significant advances in neuroimaging, allowing for subgroup identification and possible identification of potential responders, have highlighted the 
importance of this area for clinicians and researchers alike. Thus it is essential for the practicing clinician to have detailed familiarity with all of these issues.

In the clinical sphere, the neuropsychological assessment of Alzheimer's disease has also made great strides. In depth familiarity with these new developments will allow the practicing clinician to improve diagnostic accuracy.

\section{The Role of Stereotactic Surgery in Cerebral Tumors}

The course will enlighten physicians about the role of stereotactic radiosurgery in the treatment of brain tumour patients, provide them with information as to which types of brain tumour patients would be suitable for stereotactic radiosurgery, and outline stereotactic radiosurgery programs that are available in Canada.

\section{Advances in the Treatment of Multiple Sclerosis}

Multiple sclerosis (MS) is a chronic demyelinating disease primarily affecting young adults and causing both disability and economic hardship. Estimates of the prevalence of MS in Canada range from $1: 500$ to $1: 1,000$. The cause of MS remains unknown, the clinical course is quite variable, and symptomatic treatment has had limited success. Attendance at this symposium will provide participants with an increased understanding of the pathogenesis and natural history of MS, an appreciation of the role of MRI in the diagnosis and management of MS, treatment options for MS and the role of beta interferon, and an opportunity to exchange dialogue with the expert faculty.

\section{Epilepsy Rounds - Illustrated Cases}

Attendees at this course will improve their knowledge of principles of epilepsy management, gain a better appreciation of decision making in difficult cases of epilepsy, and increase their familiarity with the new antiepileptic drugs.

Sinemet CR in the Treatment of Parkinson's - Trial Results

\section{Courses}

\section{Surgery of the Occipitocervical Junction}

The course will review the anatomy, physiology and clinical biomechanics of the occipitocervical region the techniques, indications (emphasis on patient selection and alternatives) of decompression of and stabilization of the occipitocervical region. Participants will learn through case discussions the techniques and indications of occipitocervical decompression and stabilization. Participants will obtain "hands-on" experience with transoral retractor systems, $\mathrm{C} 1-\mathrm{C} 2$ stabilization techniques, and occipitocervical stabilization methods.

\section{Neurosurgery: Neuroimaging - New Concepts}

\section{Role of Endovascular Therapy in the Management of Cerebrovascular Disease}

In Section one, participants will become familiar with the role of endovascular therapy in the management of cerebrovascular disease. The course will offer didactic lectures to course participants, providing an up-to-date overview of the status of current endovascular techniques for the treatment of stroke, vasospasm, aneurysm therapy (either intra-aneurysmal coil, or proximal ballooning), arteriovenous malformations and congenital vascular malformations.

The course will review the anatomy, physiology and clinical biomechanics of the occipitocervical region the techniques, indications (emphasis on patient selection and alternatives) of decompression of and stabilization of the occipitocervical region. Participants will learn through case discussions the techniques and indications of occipitocervical decompression and stabilization. Participants will obtain "hands-on" experience with transoral retractor systems, $\mathrm{C} 1-\mathrm{C} 2$ stabilization techniques, and occipitocervical stabilization methods.

\section{New Techniques in Neurosurgery}

In section two participants will become familiar with the newer imaging techniques that may be used in neurosurgery. This course will introduce participants to Functional MRI, Positron Emission Tomography (PET), image guided neurosurgery, and CT evaluation of blood flow. The advantages and disadvantages, as well as the current and possible future uses of the techniques will be discussed during the course.

\section{Electrophysiological Studies in the Critical Care Unit}

At the end of the course, the participants should be familiar with clinical features of significant nervous system disorders encountered in the critical care unit, reasonably knowledgeable about the various electrophysiological techniques that may be utilized to investigate the central and peripheral nervous systems of patients in the critical care unit, and aware of how these electrophysiological techniques may improve treatment and determination of prognosis.

\section{Amyotrophic Lateral Sclerosis: Neurobiology to Trials - What's New?}

This course will provide the participant with an understanding of the neuropathology of ALS and the relevance of current models and neurochemical studies to this pathology; an understanding of the mechanisms of motor neuron death in ALS, and specifically the role of neurotrophic factors, excitatory amino acids and the immune system in mediating this death; an understanding of the design of therapeutic trials in ALS; and, an understanding of the basis for current therapeutic trials in ALS, and the ability to assess the therapeutic relevance of these trials.

\section{Update in Neuropathology for Neurologists and Neurosurgeons}

\section{Neurodegenerative Diseases Component}

This course will review the neuropathologic diagnoses of selected brain tumours and neurodegenerative disorders. Extensive clincopathological correlation will be provided and the audience will participate in the discussions using a "touch-pad" system. 


\section{Approaches to the History of Neuroscience}

Course attendees will gain an understanding of the various categories of medical history projects, the resources available for a neurologist or trainee who wishes to carry out a project in medical history, the various meetings, journals, and major library collections devoted to medical and neurological history, and the various on-line resources for medical and neurological history. The course will examine a history project in each category to learn how the project was carried out and the resources that were needed.

\section{Medical Management of Headache}

The course will enhance the ability of registrants to effectively treat patients with difficult migraine syndromes, effectively treat patients with chronic daily headache and medication overuse, and utilize psychologic and psychiatric resources appropriately in the management of patients with difficult headache problems. The pathophysiology of migraine, with relevance to pharmacological therapy will be discussed.

\section{Neurodegenerative Diseases of Childhood}

This course will teach an approach to the investigation and diagnosis of childhood neurodegenerative diseases. Attendees will obtain an update on new information, especially advances in genetics about specific neurodegenerative diseases from experts in the area, and will learn about possible treatments, present and future, for these conditions. Participants will the opportunity to discuss individual questions with the panel of experts.

\section{On-line Stroke Guidance System}

This presentation will demonstrate a project which will be launched this summer in Europe and Canada which provides online guidance through the maze of evolving literature on stroke and the clinical trials available. It is spearheaded by Synapse Publishing, part of the Networks of Centres of Excellence project HEALNet, and is a G7 Global Health Applications Project. Members of the Canadian Stroke Consortium have taken an active role in generation of some of the content.

It is an example of a new class of publishing called the Neural Document, literature that is dynamic and evolves over time based on feedback from use (outcome from clinical trials run through the system or from feedback from readers).

\section{Neurobiology Review Course: 1996}

\section{Alzheimer Disease - Recent Advances}

Alzheimer disease: Dr. Arthur Clark will review the current status of molecular changes in this disease; Dr. Peter St. George-

Hyslop will review the current understanding of the genetic aspects of the disease.

\section{Genetic Disorders of the Nervous System}

Genetic Diseases: Two broad classes of neurogenetic disorders will be reviewed. Dr. Dennis Bullman will present an approach to the investigation of disorders that affect single genes and Dr. George Ebers will discuss multiple sclerosis as a complex genetic trait.

\section{Modern Approaches to Pain Management}

Pain: Dr. F. A. Lenz, a neurosurgeon, will remind course attendees of the central processing of pain stimuli, an area in which he has made important contributions. Dr. A. Dray will review the basic features of peripheral pain mechanisms. Both speakers will relate their presentations to the important clinical problem of pain management.

\section{Parkinson Disease}

Parkinson Disease: Dr. Jon Stoessl will review the current understanding of dopamine receptors and how this relates to the medical treatment of Parkinson disease. As a rationale for surgical treatment of the disease, Dr. Andres Lozano will review the current understanding of basal ganglia circuitry.

\section{GENERAL INFORMATION}

Conference Site:

\section{ACCOMMODATION:}

Radisson Plaza Hotel

300 King Street

London, ON N6B 1S2

Phone (519) 439-1661

Fax (519)438-5386

\section{ADDITIONAL INFORMATION:}

Secretariat Office

Suite 810,906 - 12th Avenue SW

Calgary Alberta Canada T2R 1K7

Telephone: (403) 229-9544

Fax: (403) 229-1661;

E-Mail: brains@ccns.org
London Convention Centre 300 York Street London Ontario N6B 1 P8 (519) 661-6374

Delta Armouries Hotel 325 Dundas Street London, ON N6B IT9 Phone (519) 679-6111 Fax (519) 679-3957 